\title{
A Survey on Various Receivers for UWB Communication
}

\author{
S. Saranya Pauline ${ }^{1}$, C. Veeralakshmi ${ }^{2}$ \\ ${ }^{I}$ (PG student, Department of ECE, M. Kumarasamy College of Engineering, Karur, Tamilnadu, India) \\ ${ }^{2}$ (Assistant Professor, Department of ECE, M. Kumarasamy College of Engineering, Karur, Tamilnadu, India)
}

\begin{abstract}
Ultra Wideband (UWB) is an inherent technology used in current wired and wireless communication systems. These systems share the radio frequency spectrum with narrowband signals and also provide high data rates, low cost, greater bandwidth, good time domain resolution and improved channel capacity. UWB technology has attracted a lot of inquisitiveness in researchers worldwide. There are several challenges in designing a UWB receiver such as channel estimation and interference mitigation. In multi-user environments, multiple access interference (MAI) degrades the performance of UWB systems. To prevail over these challenging issues, an adaptive and robust receiver needs to be designed to alleviate interference in all types of environments. In this paper, the different types of receiver structures like Rake, energy, correlation, suboptimal, near-optimal and adaptive nonlinear rake receivers are surveyed and their performances are analyzed. This survey helps to realize superior bit error rates (BER) and excellent signal-to-noise ratios (SNR).
\end{abstract}

Keywords: Adaptive nonlinear rake receivers, Correlation receivers, Energy detectors, Rake receivers, Ultra wideband (UWB).

\section{Introduction}

The volatile escalation of the wireless communication market is liable to prolong in the future, as the demand for all types of wireless services is growing. New generations of wireless mobile radio systems aims to offer flexible data rates and a wide variety of applications to the mobile users while serving as many users as possible. This objective, nevertheless, needs to be achieved using limited available resources like spectrum and power. As more and more devices go wireless, future technologies will face spectral crowding and hence coexistence of wireless devices will be a foremost issue. Taking into consideration the inadequate bandwidth availability, accommodating the demand for higher capacity and data rates becomes a challenging task that requires innovative technologies which can coexist with devices operating at various frequency bands. Recently, UWB technology has shifted its focus on consumer electronics and communications. Ideal targets for UWB systems are low power, low cost, high data rates, precise positioning capability, and extremely low interference.

UWB systems are based on impulse radio concepts. Impulse radio generates a sequence of miniature interval pulses, of the order of hundreds of picoseconds. Each pulse has a very wide spectrum, which adheres to the spectral mask needs [1]. UWB is acknowledged as Radio frequency (RF) technology that transmits binary data in the form of short impulses over an ample range of frequencies in the range of 3.1 to $10.6 \mathrm{GHz}$ [2]. These systems offer advantages like improved channel capacity, low power transmission, low power dissipation, low power spectral density, high security and low cost. The diverse application areas include radar, aviation, intrusion detection in sensor networks, high precision location navigation systems and high speed LAN/WAN. UWB technology also finds uses in location and tracking applications due to its very good time domain resolution features. Narrow time domain pulses allow UWB radios to offer timing precision superior than GPS (global positioning system) and other radio systems.

UWB uses low-energy and extremely short duration pulses or bursts of RF (radio frequency) energy over a wide spectrum of frequencies, to transmit data over short to medium distances, say about 15 to100 m. It does not use a carrier wave to transmit data. It has a bandwidth greater than $500 \mathrm{MHz}$ and occupies $20 \%$ of centre frequency according to Federal Communications Commission (FCC) [2]. In the mixer, a baseband signal is taken and a carrier frequency is injected or translated into a signal of frequency which has enviable proliferation characteristics. The wideband temperament of the UWB signal allows it to span the carrier frequencies. The UWB signal propagates well exclusive of additional up-conversion and amplification process. Up conversion is not required in the UWB receiver. Hence local oscillator, associated complex delay and phase tracking loops are absent in the receiver. Very high multipath resolution is achieved as a result of the large bandwidth of the transmitted signal [1].

UWB systems have noise-like signals that are defiant to rigorous multipath and jamming. But due to the low power nature of the noise-like signals, UWB transmissions do not cause significant interference to existing radio systems. This interference fact between impulse radio and the other existing radio systems is one of the significant topics in present UWB research. UWB uses extremely wide bandwidth, so if the emission power is not well controlled, then these devices might cause interference with other existing systems. UWB systems are categorized as an expansion of conventional spread spectrum (SS) systems. One of the major 
contradictions between UWB systems and conventional SS systems is the radio channel which they use. The UWB channel is enormously rich in multipath. The combined multipath components increase the total signal power. The uncombined multipath component causes interference. If there is notably greater number of resolvable multipath components, more receiver elements need to be taken into account. In multi user environments, multiple access interference (MAI) significantly degrades the performance of these UWB systems.

\section{UWB SYSTEM MODEL}

UWB uses a kind of pulse modulation. The Gaussian pulse is the most widely used pulse in UWB systems. A UWB transmitter emits a single and short time domain sine wave pulse (called a monocycle) which propagates devoid of an additional RF (radio frequency) mixing stage. This monocycle has no data in it. It is the timing between monocycle that determines whether 0 or 1 is transmitted. The duration of the short pulse is generally less than 1 nanosecond. The two basic types of modulation techniques in UWB systems are timebased techniques and shape-based techniques and is shown in Fig. 2. The commonly used time- based method is pulse position modulation (PPM) where each pulse is delayed or sent in advance. The other shape based method is to create a pulse with opposite phase. This is known as bi-phase modulation (BPM). Orthogonal pulse modulation (OPM) requires special pulse shapes to be generated which are orthogonal to each other. On-Off keying (OOK) requires the information about the absence or presence of a pulse which signifies the digital information of ' 0 ' or ' 1 ' respectively. Pulse amplitude modulation (PAM) is a technique where the amplitude of the pulse is varied to contain digital information.

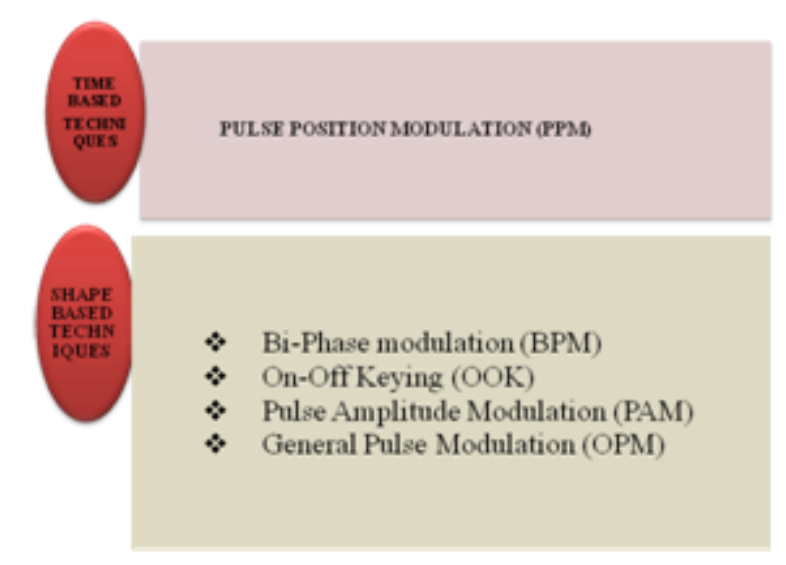

Fig. 2 Division of different modulation methods for UWB communications

In the transmitter as shown in Fig. 3, meaningful data is generated by applications that are quite separate from the physical layer transmitter. This binary information stream is conceded to the front end to map from bits to symbols. These symbols are then mapped to an analog pulse shape. Pulse shapes are generated by the pulse generator. Precise timing circuitry is required to send the pulses out at intervals which are meaningful.

The receiver as shown in Fig. 4 recovers the data and passes the data to back end. There are two major differences between the transmitter and the receiver. The first is that the receiver will have an amplifier to boost the signal power of the received weak signals. The second is that the receiver must detect to locate the required pulses amongst the other signals and then track these pulses to compensate for any mismatch between the clocks of the transmitter and the receiver.

The optimal receiver technique used in UWB is a correlation receiver. A correlator multiplies the received RF signal by a template waveform and then integrates the output of that process to yield a single DC voltage. The wireless channel suffers from multipath, where reflections and other effects of the channel cause multiple copies of the transmitted pulse to appear at the receiver. If a rake receiver is used, these extra pulses can be used to improve reception but at the cost of increased receiver complexity.

When a monocycle is buried in the noise of other signals, it becomes difficult to detect a single UWB pulse. However, by adding together multiple correlator samples known as pulse integration, the signals can be transmitted. Through pulse integration, UWB receivers can acquire, track, and demodulate UWB transmissions that are significantly below the noise floor. 


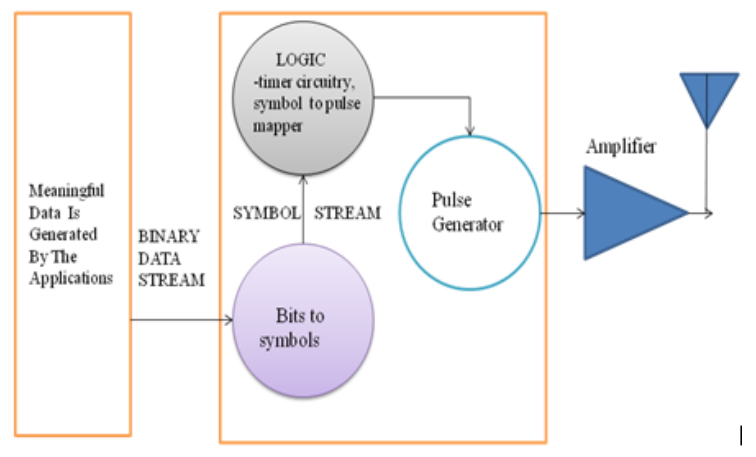

Fig: 3. UWB transmitter

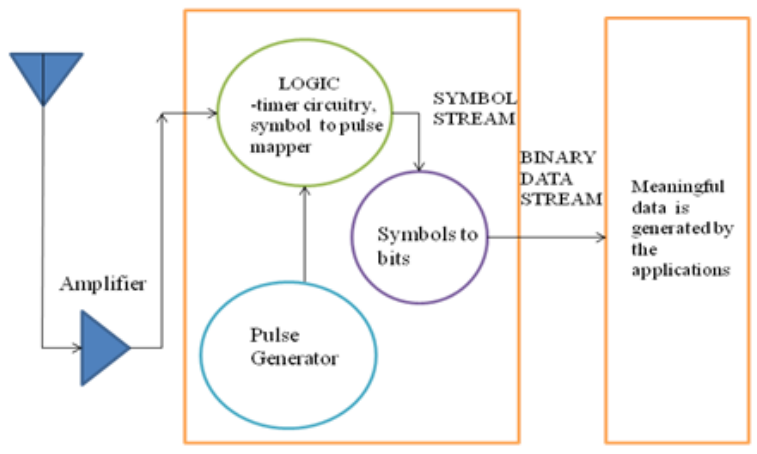

Fig: 4. UWB receiver

\section{RECEIVER CATEGORIES}

The system model of UWB shows that the design of a transmitter is much less intricate compared to the receiver. The various design challenges for a UWB receiver are its intricacy, coexistence with other services, managing strong narrowband interference, shaping spectrum of transmitted signals, precise synchronization and channel parameter estimation, potent dispensation capabilities for high performance and lucid digital receiver structures and accurate modelling of the ultra wideband channel in assorted environments. In this section, the existing receiver structures are analysed and discussed.

\section{Low complexity Rake Receiver}

A Rake receiver is one of the simplest receiver structures to avoid multi-path fading. It combines different signal components that have propagated through the channel by different paths. The combination of different signal components will increase the signal-to-noise ratio (SNR) and also improve link performance. Generally, there are three types of rake receivers namely A-Rake, P-Rake, S-Rake. P-Rake combines the first arriving multipath components and S-rake combines the instantaneously strongest multipath components.

Whilst being much less complex, the S-rake and P-rake are not able to combine all the received signal energy and so have substantially worse performance than the A-rake. For transmission bandwidth less than 7.5 GHz, P-Rake is a good option whereas for larger bandwidth S-rake is the best [4].The general composition of a Rake receiver is shown in Fig 5. R. Malhotra described rake receiver as a radio receiver to avoid multipath fading [3]. The main shortcoming is the need for a countless number of rake branches, which also accounts for countless number of correlators.

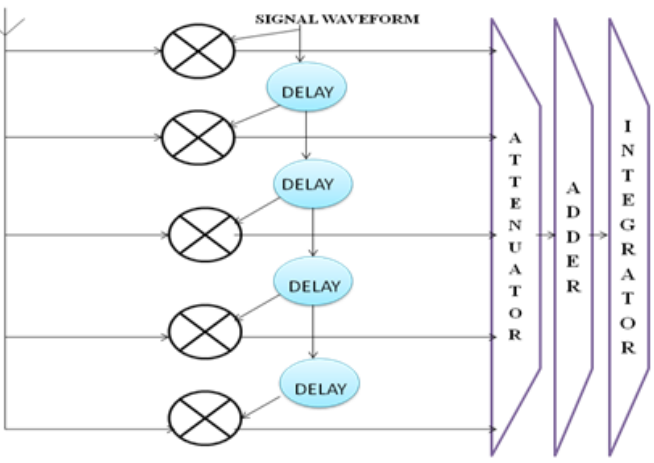

Fig: 5 General structure of a rake receiver 


\section{Rake receiver using Matched filter with three Combining techniques}

The various combining techniques used at the receiver are maximum ratio combining (MRC), equal gain combining (EGC) and selective combining (SC). A wireless rake receiver is improved for use in indoor environments with two channel modes CM3 (0 to $10 \mathrm{~m}$ ) and CM4 (4 to $10 \mathrm{~m}$ ). The received signal is the sum of the desired signal and noise. The signal is transmitted using DS-BPSK and then AWGN is added to it. A P-rake receiver receives the signals from the five stages of the matched filter and then combined using MRC, EGC and SC. From the simulation results, it is seen that MRC rake receiver structure produces better BER and SNR performance. MRC also captures much of the received signal energy. Rake receiver introduces inter symbolinterference (ISI) which is mitigated using a matched filter. But this technique can be used for indoor applications in the range of up to 10 meters only [4].

\section{Energy Detectors Receivers}

In high data rate UWB systems, ISI is a complicating factor. To suppress this ISI, an on-off keying (OOK) based low complexity energy detector with decision feedback equalization algorithm is used. Channel is supposed to be time-varying and the filter coefficients are constant. Sequences of training symbols are sent between the packets to estimate the decision feedback filter coefficients. This detector is implemented by taking the square of the received signal (using a square law device), integrating it and then passing it to a decision feedback equalizer. The advantage is that error does not propagate to more than one subsequent symbol and hence there is no error propagation. High gains are exhibited here and ISI is cancelled. The disadvantage in this approach is that energy detectors achieve low complexity at the expense of partially degraded performance [5].

\section{MAI resilient UWB W-PAN receivers}

New robust receiver structures are designed suited for ultra wide band (UWB) standard IEEE 802.15.4a to suppress multiuser access interference (MAI) in wireless personal area networks (W-PAN). Both demodulation and decoding approaches are optimized to mitigate MAI. Gaussian mixture (GM) model is used to sample the baseband signal at the receiver input and thus deriving maximum likelihood (ML) receiver.

The GM model is extended to Generalized Gaussian model (GGM) and Laplacian model. GGM receivers with $\mathrm{K}=2$ is labeled as $\mathrm{B} 4$ provides a notable gain of $10 \mathrm{~dB}$ with PER of $10^{-4}$. All these algorithms have low PER performance in office environments and accomplish better only in indoor environments [6].

\section{Hybrid Matched Filter Correlation Receivers}

A hybrid matched filter Transmitted-reference (TR) correlation receiver is used to perform matched filter detection before correlating the data symbol with the reference symbol. TR schemes permit the receiver structures to combine energy from various multipath components exclusive of channel estimation and also it requires only frame-rate sampling. Noise- noise cross terms effects are analyzed and reduced using a matchedfilter operation.

BPSK modulation scheme is used and hence SNR is much better. The correlated signals are integrated to capture signal energy from the impulse response. If the interference between the reference and data pulse is larger than the maximum delay, then processing gain is restricted to an extent. An analog correlator is needed for the time hopping sequence of the pulses [7].

\section{Space time S-Rake Receiver with four FSSs}

A space-time S-Rake receiver with minimum signal-to-interference-plus-noise ratio (MSINR) is used with multiple antennas in direct sequence (DS) UWB systems for indoor applications. Four finger selection strategies (FSS) namely optimum FSS (Opt-FSS), constrained energy-based FSS (CEB-FSS), hybrid energy-based FSS (HEB-FSS) and energy based FSS (EB-FSS). Opt-FSS achieves best performance but involves great complexity. EB-FSS is suitable for weak narrow band interference (NBI) and CEB-FSS is suitable for strong NBI. HEB-FSS is suitable for a variety of NBI and offers the best performance of all in terms of NBI suppression [8].

\section{Near-optimal and Suboptimal receivers}

For dense multipath channels, non-Gaussian multiuser interference (MUI) at each finger of a rake receiver is considered. Near-optimal (NO) and Sub-optimal (SO) receivers are designed for these multiuser UWB-IR systems. User's path gain and MUI statistics need to be estimated.

MUI statistics are evaluated using second and fourth order moments. MUI is modeled by a generalized Gaussian distribution. IEEE 802.15.3a channel model 1 (CM1) are used for simulation. NO receiver is significant in the range of $\mathrm{K}=2$ to 10 in indoor communications. For small number of users, diversity gain is significant. For higher data rates an optimal receiver needs to be designed while keeping in mind the MUI statistics [9]. 


\section{Adaptive Nonlinear Rake Receivers}

An alpha-stable distribution modeling is done for the interference plus noise in the environment. An adaptive nonlinear rake receiver robust to changes in user density and noise level is studied for use in UWB multiple access systems in order to suppress MAI. The main obstacle here is to determine the alpha stable parameters accurately. A myriad filter is used for detection purposes and it helps to estimate the location parameters of an alpha-stable process. A myriad filter estimates the location parameter based on observations in which a suitable value of $\mathrm{K}$ is chosen. $\mathrm{K}$ is known as tuning or linearity parameter. BER performance is improved only in the high SNR region and not in the low SNR region. In the low SNR region, the total additive noise is less impulsive due to the dominance of Gaussian noise [10].

\section{CONCLUSION}

This survey has outlined the UWB concepts, various modulation and reception techniques in an UWB system model. UWB systems require robust and adaptive receiver designs that are less complex. It should squander less power and also have the ability to alleviate any type of interference in all environments. UltraWideband Receiver is thus analyzed as a medium of communication in various wired and wireless communication applications due to its innate valuable factors. The different UWB receiver designs are analyzed using performance metrics like BER. On analysis, it is found that the adaptive nonlinear rake receiver provides higher BER and SNR values than the other conventional receivers. Adaptive rake receivers also reduce nonGaussian multiple access interference to an elevated degree via a myriad filter approach.

\section{REFERENCES}

[1] Ian Oppermann, Matti Hamala Inen and Jari Iinatti., UWB theory and Applications (Wiley, 2004).

[2] R. Malhotra, "UWB Communication Receiver: Review and Design Considerations", Int. J. of Advanced Engineering Sciences and Technologies, vol. No. 8, no. 2,197-202.

[3] D. Cassioli, M.Z. Win, F. Vatalaro, A.F. Molisch, "Low Complexity Rake Receivers in Ultra-Wideband Channels", IEEE Trans. Wireless Comm. vol. 6, no.4, April 2007.

[4] Rashid A. Fayadh, F. Malek, Hilal A. Fadhil, N. A. Mohd Affendi, Azuwa Ali and M. F. Haji Abd Malek "Adaptive Rake Receiver Using Matched Filter with Three Combining Techniques", Australian J. of Basic and Applied Sciences, 7(5), 26-33, 2013.

[5] Mustafa E. S, Ahin and Huseyin Arslan "Inter-symbol Interference in High Data Rate UWB Communications Using Energy Detector Receivers", Research by Honeywell, Inc. and Custom manufacturing \& Engineering, Inc.

[6] Tomaso Erseghe and Stefano Tomasin "Optimized Demodulation for MAI Resilient UWB W-PAN Receivers “, IEEE 2008.

[7] Fredrik Tufvesson, Sinan Gezici, and Andreas F. Molisch "Ultra-Wide band Communications using Hybrid Matched Filter Correlation Receivers", IEEE Trans. Wireless Comm.., vol. 5, no. 11, Nov. 2006.

[8] Tsung-Hui Chang, Chong-Yung Chi and Yu-Jung Chang "Space-Time Selective RAKE Receiver With Finger Selection Strategies for UWB Overlay Communications", IEEE Transactions on Microwave theory and techniques, vol. 54, no. 4, April 2006.

[9] Dong In Kim "Near-optimal and Suboptimal Receivers for Multiuser UWB Impulse Radio Systems in Multipath", IEEE Trans. Comm., vol. 57, no.11, November 2009.

[10] S. Niranjayan and N.C. Beaulieu "Novel Adaptive Nonlinear Receivers for UWB Multiple Access Communications", IEEE Trans. Wireless Comm.., vol. 12, no.5, May 2013. 